04

\title{
Аналитический расчет распределений электронной плотности и концентрации ионов примеси в термической пылевой плазме с применением модели „желе“ для конденсированных частиц
}

\author{
(С) И.И. Файрушин ${ }^{1}$, И.Г. Даутов ${ }^{1,2}$, Н.Ф. Кашапов ${ }^{1}$, \\ А.Р. Шамсутдинов ${ }^{1}$ \\ ${ }^{1}$ Казанский федеральный университет \\ ${ }^{2}$ Казанский национальный исследовательский технический университет \\ им. А.Н. Туполева - КАИ \\ E-mail: wolobokr@gmail.com
}

Поступило в Редакцию 5 июля 2016 г.

С применением модели „желе“ к описанию частиц конденсированного вещества получены самосогласованные пространственные распределения электронной плотности во всем объеме частиц и окружающей их плазме, а также распределения концентрации ионов легкоионизирующихся примесных атомов. Установлено, что в термической пылевой плазме, содержащей примесь легкоионизирующегося элемента, при повышении температуры эмиссия электронов конденсированными частицами может ослабевать. Показано, что с уменьшением радиуса частиц при постоянной температуре эмиссия ими электронов увеличивается. Вблизи поверхности конденсированных частиц отмечается образование области плазмы с нарушением ионизационного равновесия.

Систему, состоящую из частично ионизованного газа и частиц конденсированного вещества микронных размеров, принято называть пылевой плазмой или плазмой с конденсированной дисперсной фазой (КДФ) [1]. Одной из разновидностей этого вида плазмы является термическая плазма, в которую вводят или же в ней образуются частицы микронных размеров [2-6]. Такая система, например, формируется при сгорании металлических частиц, сопровождающемся образованием оксидов металлов в форме микронных частиц [6,7]. Это связано с тем, что при температуре горения происходит тепловая ионизация 
конденсированной и газовой сред, которая затрагивает все этапы становления и роста конденсированных частиц [7].

Исследования термической плазмы, содержащей заряженные микрочастицы конденсированного вещества, также актуальны в связи с возникновением такой плазмы в процессах получения функциональных покрытий плазменным напылением [8]. Необходимо отметить, что, так как термическая пылевая плазма (ТПП) является дисперсной системой, огромную роль в проявлении разнообразия ее свойств играют процессы, происходящие у поверхности конденсированных частиц. Таким образом, для описания данной системы необходимо привлекать и методы физики поверхности. На сегодняшний день в физике поверхности получено много результатов, описывающих электронные свойства поверхности веществ [9-15]. Рассмотрены также размерные зависимости различных параметров, а также влияние окружающей среды на свойства поверхности. Например, исследованы снижение работы выхода электрона из металла, покрытого диэлектрическими алмазоподобными, полимерными, оксидными пленочными покрытиями [9-11], а также влияние окружающей среды на свойства поверхности. При рассмотрении вопроса об описании распределения потенциала электрического поля внутри конденсированной среды и у ее поверхности широкое применение нашла модель „желе“, согласно которой ионный остов конденсированной среды представляется в виде непрерывного однородного положительного фона, а электроны проводимости как электронный газ внутри этого положительного фона. Данный вопрос подробно рассматривался, например в [9-14]. Применительно к малым частицам эта задача была рассмотрена в работах [16-19], в которых при описании малых частиц также использована модель ,желе“.

Проблема определения распределения потенциала электрического поля также занимает одно из центральных мест в теоретическом описании ТПП. В ряде работ [3-5] по исследованию эмиссионной зарядки пылевых частиц в газе и плазме получены расчетные данные по распределению потенциала и электронной плотности в пространстве между частицами. Однако вопрос распределения потенциала и электронной плотности внутри самих частиц применительно к пылевой плазме обходится стороной, а заряд частиц определяется уравнением баланса с помощью формулы Ричардсона-Дешмана, в которой значение работы выхода электронов считается зависящим только от материала

Письма в ЖТФ, 2016, том 42, вып. 23 
частицы. Однако работа выхода зависит, кроме того, от радиуса частицы и ее температуры, что показано, в частности, в работе [20].

В данной работе решается задача объединенного описания распределения потенциала электрического поля для областей внутри пылевых частиц и окружающей их плазмы. Рассматриваются полупроводниковые частицы, в этом случае плотность положительного фона определяется концентрацией атомов, электроны которых способны свободно перемещаться внутри частицы. Температура компонент системы „пылевые частицы-плазма“ считается одинаковой, что реализуется в ряде случаев при атмосферном или более высоком давлениях [2].

Сделаем допущение, что электронный газ внутри частицы, как и в окружающей плазме, имеет концентрацию ниже порога вырождения, поэтому к нему можно применить распределение Больцмана

$$
n_{e}=n_{e 0} e^{\frac{q \varphi}{k T}}
$$

где $n_{e}-$ электронная плотность, $q-$ абсолютное значение заряда электрона, $\varphi-$ потенциал электрического поля, $n_{e} 0-$ электронная плотность при $\varphi=0, k$ - постоянная Больцмана, $T-$ абсолютное значение равновесной температуры всех компонент системы. В данной работе рассматривается диапазон изменения $T$ от 2000 до $2500 \mathrm{~K}$, характерный для ТПП [2-6]. В качестве значений плотности положительного фона частицы и относительной диэлектрической проницаемости материала частицы в расчетах взяты соответственно значения $n_{i}^{(1)}=10^{21} \mathrm{~m}^{-3}$ и $\varepsilon=10$.

Когда газ, окружающий пылевые частицы, не содержит легкоионизирующихся примесей, ионизацией самого газа в рассматриваемом диапазоне температур можно пренебречь. В данной же работе примем, что в газе находится примесь легкоионизирующегося щелочного металла. Для определения концентрации заряженной компоненты в окружающей пылевые частицы плазме воспользуемся известным уравнением Саха

$$
\frac{n_{i}^{(2)} n_{e}}{n_{a}}=\frac{2 z_{i}}{z_{a}}\left(\frac{2 \pi m_{e} k T}{h^{2}}\right)^{\frac{3}{2}} e^{\frac{q \varphi_{u}}{k T}}
$$

где $n_{i}^{(2)}, n_{a}-$ концентрация ионов и атомов примеси соответственно, концентрация электронов (электронная плотность), $z_{i}, z_{a}-$ статистические суммы ионов и атомов примеси соответственно (в расчетах 
далее используются значения, соответствующие калию), $m_{e}-$ масса электрона, $h$ - постоянная Планка, $\varphi_{u}-$ потенциал ионизации примесного атома.

В рассматриваемом диапазоне температур частицы будут эмитировать в окружающие их плазму электроны и приобретать некоторый положительный заряд. Таким образом, вокруг пылевой частицы образуется электрическое поле, которое будет влиять на пространственное распределение концентраций электронов и ионов у поверхности частицы. Для простоты будем считать, что частицы имеют форму шара одного радиуса $R$ и равномерно распределены в пространстве с расстоянием между центрами $2 l$.

Как известно, связь потенциала электрического поля и концентрации электрических зарядов задается уравнением Пуассона. В представленном случае, когда электронный газ во всем объеме ТПП невырожден, данное уравнение будет являться уравнением Пуассона-Больцмана. Учитывая то, что рассматриваемая задача является сферически симметричной и ионы примеси в плазме имеют больцмановское распределение, окончательно получим

$$
\begin{aligned}
\frac{d^{2} \varphi}{d r^{2}} & +\frac{2}{r} \frac{d \varphi}{d r}=\frac{q}{\varepsilon_{0}}\left(\frac{n_{e 0} e^{\frac{q \varphi}{k T}}}{(\varepsilon-1) \theta(R-r)+1}\right. \\
& \left.-\frac{n_{i}^{(1)}}{\varepsilon} \theta(R-r)-n_{i 0}^{(2)} e^{-\frac{q \varphi}{k T}} \theta(r-R)\right),
\end{aligned}
$$

где $r$ - координата, отсчитываемая от центра частицы, $\varepsilon_{0}-$ электрическая постоянная, $\theta(r)-$ функция Хевисайда, $n_{i 0}^{(2)}-$ концентрация ионов примеси при $\varphi=0$. За начало отчета потенциала примем поверхность частицы, т. е. $\varphi(R)=0$. Задача симметрична относительно центра частицы, поэтому $\varphi^{\prime}(0)=0$. К задаче необходимо применить еще и условие равенства нулю суммарного заряда в объеме, приходящемся на одну частицу, т.е. $\varphi^{\prime}(l)=0$. В случае $|q \varphi| \ll k T$ экспоненциальные множители в правой части уравнения (2) можно разложить в ряд и ограничиться первыми двумя членами, тогда данное уравнение решается аналитически. Далее будут рассмотрены только те решения, которые достаточно хорошо удовлетворяют данному условию. Решение произведено в два этапа: сначала рассматривается область $0 \leqslant r<R$, а затем, принимая во внимание условия непрерывности электронной

Письма в ЖТФ, 2016, том 42, вып. 23 
плотности и электрического поля на поверхности частицы, получается распределение потенциала и в области $R \leqslant r \leqslant l$. Аналитическая запись выражений, описывающих распределение потенциала, будет следующей:

$$
\begin{gathered}
\psi(x)=\left(\bar{n}_{i}^{(1)}-1\right)\left(\frac{e^{-a x}-e^{a x}}{x\left(e^{a}-e^{-a}\right)}+1\right) \text { для } 0 \leqslant x<1, \\
\psi(x)=\frac{1}{\bar{n}_{i 0}^{(2)}+1}\left(\frac{C_{2} e^{b x}+C_{3} e^{-b x}}{x}+\bar{n}_{i 0}^{(2)}-1\right) \text { для } 1 \leqslant x \leqslant \lambda .
\end{gathered}
$$

Здесь

$$
\begin{gathered}
\psi=\frac{q \phi}{k T} ; \quad \bar{n}_{i}^{(1)}=\frac{n_{i}^{(1)}}{n_{e 0}} ; \quad x=\frac{r}{R} ; \quad a=q R \sqrt{\frac{n_{e 0}}{\varepsilon \varepsilon_{0} k T}} \\
\bar{n}_{i 0}^{(2)}=\frac{A n}{n_{e 0}^{2}+A n_{e 0}} ; \quad A=\frac{2 z_{i}}{z_{a}}\left(\frac{2 \pi m_{e} k T}{h^{2}}\right)^{\frac{3}{2}} e^{\frac{q \varphi_{u}}{k T}}
\end{gathered}
$$

$n=n_{a}+n_{i 0}^{(2)}-$ концентрация атомов примеси до ионизации, а $n_{a}-$ после ионизации; $b=a \sqrt{\varepsilon\left(1+\bar{n}_{i 0}^{(2)}\right)} ; \lambda=\frac{l}{R}$. Кроме того,

$$
\begin{gathered}
\bar{n}_{i}^{(1)}=1-C_{1}\left(e^{a}-e^{-a}\right) ; \quad C_{1}=\frac{C_{2}-(b+1)\left(1-\bar{n}_{i 0}^{(2)}\right)}{\left(\bar{n}_{i 0}^{(2)}+1\right)\left(e^{a}(a-1)+e^{-a}(a+1)\right)} \\
C_{2}=\frac{(b \lambda+1)\left(1-\bar{n}_{i 0}^{(2)}\right) e^{b(1-2 \lambda)}}{(b \lambda+1) e^{b(1-2 \lambda)}+b \lambda-1} ; \quad C_{3}=e^{b}\left(1-\bar{n}_{i 0}^{(2)}-C_{2} e^{b}\right)
\end{gathered}
$$

Зная распределение потенциала, через формулу (1) несложно определить распределения электронной плотности и концентрации ионов примеси. На рис. 1 приведен график распределения концентраций электронов и ионов в области $0 \leqslant r \leqslant l$.

Как видно из рис. 1, с повышением температуры величина электронной плотности на всем интервале от центра частицы до $r=l$ растет. Рост электронной плотности в плазме, очевидно, объясняется повышением ее степени ионизации при увеличении температуры. Внутри же частицы плотность электронов с повышением температуры должна была бы понизиться, так как в этом случае следовало бы ожидать увеличения эмиссии электронов, как это происходит, когда ионизация

Письма в ЖТФ, 2016, том 42, вып. 23 


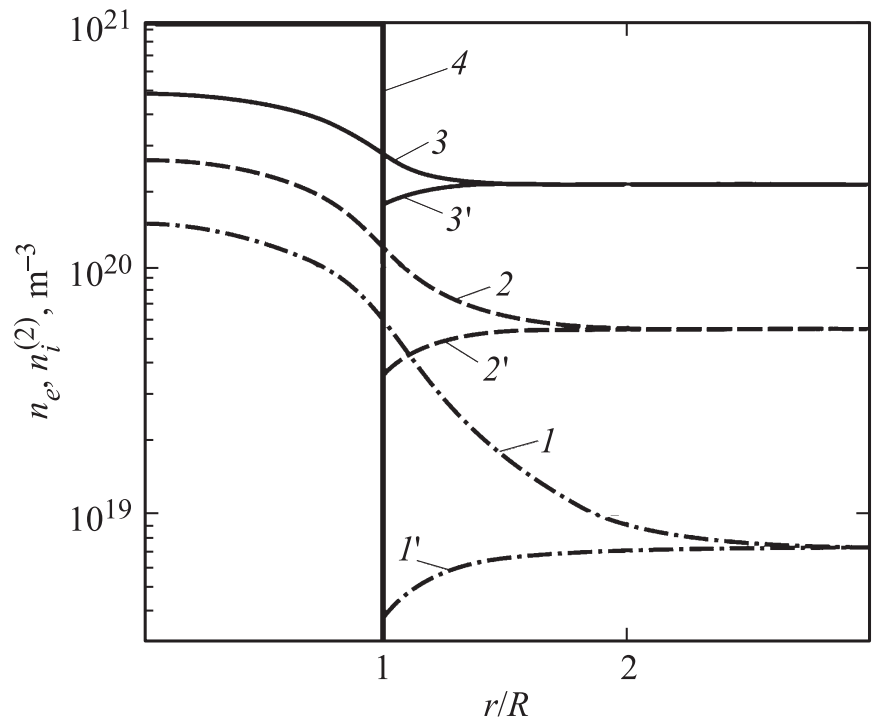

Рис. 1. Распределения электронной плотности (кривые $1,2,3$ ) и концентрации ионов примеси (кривые $1^{\prime}, 2^{\prime}, 3^{\prime}$ ) при $n=10^{23} \mathrm{~m}^{-3}, R=10^{-6} \mathrm{~m}, l=3 R$ и различных значениях температуры ( 1 и $1^{\prime}$ при $T=2000 \mathrm{~K}, 2$ и $2^{\prime}$ при $T=2250 \mathrm{~K}, 3$ и $3^{\prime}$ при $\left.T=2500 \mathrm{~K}\right)$; кривая 4 соответствует величине $n_{i}^{(1)}$.

газа, окружающего частицу, пренебрежимо мала [21]. В рассматриваемом случае выходу электронов из частиц начинает препятствовать увеличивающаяся концентрация электронов самой плазмы, возникающих благодаря процессу ионизации атомов примеси. При понижении температуры видно, что увеличение концентрации свободных электронов в плазме происходит в основном за счет электронной эмиссии из конденсированных частиц, а ионизация атомов примеси достаточно низкая (кривые 1 и $1^{\prime}$ ).

Также наблюдается изменение характера пространственного распределения электронной плотности в зависимости от радиуса частицы (рис. 2). С уменьшением размера частиц увеличивается выход электронов из частицы в окружающую плазму. Это объясняется увеличением удельной поверхности с уменьшением радиуса частиц. Таким образом,

Письма в ЖТФ, 2016, том 42, вып. 23 


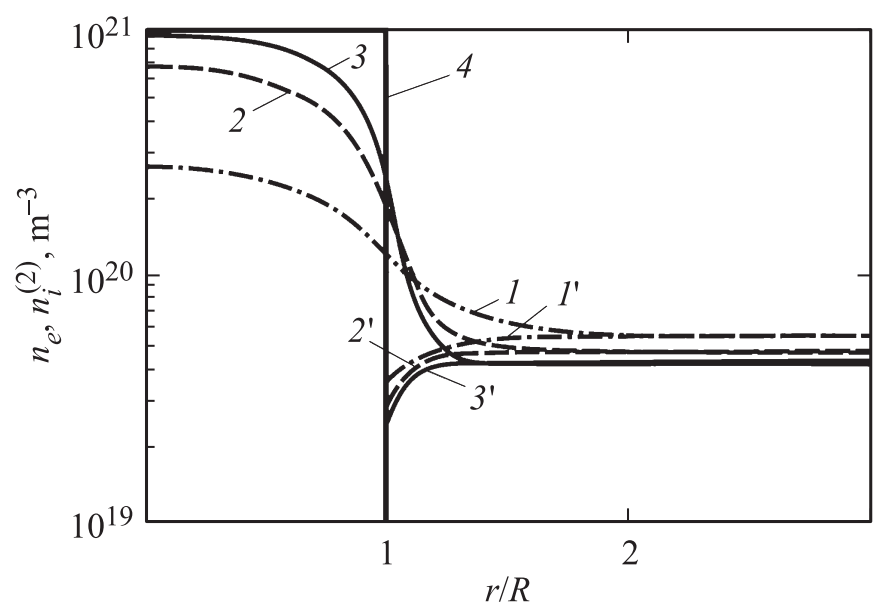

Рис. 2. Распределения электронной плотности (кривые $1,2,3)$ и концентрации ионов примеси (кривые $1^{\prime}, 2^{\prime}, 3^{\prime}$ ) при $n=10^{23} \mathrm{~m}^{-3} ; T=2250 \mathrm{~K} ; l=3 R$ и различных значениях радиуса частиц ( 1 и $1^{\prime}$ при $R=10^{-6} \mathrm{~m}, 2$ и $2^{\prime}$ при $R=2 \cdot 10^{-6} \mathrm{~m}, 3$ и $3^{\prime}$ при $\left.R=3 \cdot 10^{-6} \mathrm{~m}\right)$; кривая 4 соответствует величине $n_{i}^{(1)}$.

частицы меньших размеров более эффективно эмитируют электроны в окружающую плазму.

Нужно заметить, что у поверхности частицы ионизационное равновесие нарушается. На больших расстояниях от частицы концентрации ионов и электронов выравниваются, плазма здесь квазинейтральная. Нарушение ионизационного равновесия более отчетливо наблюдается при уменьшении температуры плазмы и радиуса частиц.

Следует отметить, что решенная в данной работе задача подобна той, которая возникает при определении распределения концентрации электронов в контакте двух полупроводников $n$-типа с разной концентрацией свободных носителей заряда (в данном случае электронов) [см., например, 22]. Разница, по существу, состоит в том, что в нашем случае, когда рассматривается контакт равновесной плазмы с полупроводником, носители положительного заряда со стороны плазмы подвижны и испытывают влияние от возникающего вследствие перераспределения зарядов электрического поля.

Письма в ЖТФ, 2016, том 42, вып. 23 
В заключение можно сказать следующее, подбирая параметры термической плазмы, окружающей конденсированные частицы, появляется возможность влиять на величину и распределение электронной плотности в пространстве между пылевыми частицами, а значит, и на электропроводность пылевой плазмы в целом. Это важно для ряда приложений, таких, например, как получение покрытий плазменным напылением и плазмохимический синтез дисперсных материалов.

Работа выполнена за счет средств субсидии, выделенной в рамках государственной поддержки Казанского федерального университета в целях повышения его конкурентоспособности среди ведущих мировых научно-образовательных центров.

\section{Список литературы}

[1] Фортов В.Е., Храпак А.Г., Якубов И.Т. Физика неидеальной плазмы: Учебное пособие. М.: Физматлит, 2004.

[2] Vishnyakov V.I. // Phys. Rev. E. 2012. V. 85. P. 026402.

[3] Фортов В.Е., Филинов В.С., Нефедов А.П. и др. // ЖЭТФ. 1997. В. 111. C. 889.

[4] Дьячков Л.Г., Храпак А.Г., Храпак С.А. // ЖЭТФ. 2008. В. 133. С. 197.

[5] Khrapak S.A., Morfill G.E., Fortov V.E. et al. // Phys. Rev. Lett. 2007. V. 99. P. 055003.

[6] Драган Г.С. // ЖЭТФ. 2004. В. 125. С. 507.

[7] Золотко А.Н., Полетаев Н.И., Вовчук Я.И. // ФГВ. 2015. В. 2. С. 125.

[8] Пузряков А.Ф. Теоретические основы технологии плазменного напыления. М.: МГТУ им. Н.Э. Баумана, 2003.

[9] Юмагузин Ю.М., Корнилов В.М., Лачинов А.Н. // ЖЭТФ. 2006. В. 130. C. 303.

[10] Лошищкая К.П., Созаев В.А., Чернышова Р.А. // Поверхность. 2005. В. 9. C. 104.

[11] Смогунов А.Н., Куркина Л.И., Фарберович О.В. // ФТТ. 2000. В. 42. С. 1848.

[12] Коротков П.К., Созаев В.А., Тхакахов Р.Б., Уянаева З.А. // Изв. РАН. Сер. Физ. 2009. В. 73. С. 1038.

[13] Мамонова М.В., Прудников В.В., Прудникова И.А. Физика поверхности. Теоретические модели и экспериментальные методы. М.: Физматлит, 2011.

[14] Ролдугин В.И. Физикохимия поверхности. Долгопрудный: Интеллект, 2011.

[15] Партенский М.Б. // УФН. 1979. В. 128. С. 69.

[16] Партенский М.Б. // Поверхность. 1982. В. 10. С. 15.

[17] Смирнов М.Б., Крайнов В.П. // ЖЭТФ. 1999. В. 115. С. 2014.

4 Письма в ЖТФ, 2016, том 42, вып. 23 
[18] Ekardt W. // Phys. Rev. B. 1984. V. 29. P. 1558.

[19] Иванов В.К., Ипатов А.Н. // ЖЭТФ. 1996. В. 109. С. 902.

[20] Смирнов Б.М. // УФН. 2000. В. 170. С. 495.

[21] Dautov G., Fayrushin I., Dautov I., Kashapov N. // J. Phys.: Conf. Ser. 2013. V. 479. P. 012014

[22] Бонч-Бруевич В.Л., Калашников С.Г. Физика полупроводников. М.: Наука, 1977. 